\title{
¿Simple Analytic Solutions for a Convectively Driven Walker Circulation and Their Relevance to Observations
}

\author{
JUHO IIPPONEN ${ }^{\mathrm{a}}$ AND LEO DONNER ${ }^{\mathrm{b}}$ \\ ${ }^{a}$ Program in Atmospheric and Oceanic Sciences, Princeton University, Princeton, New Jersey \\ ${ }^{\mathrm{b}}$ NOAA/Geophysical Fluid Dynamics Laboratory, Princeton, New Jersey
}

(Manuscript received 15 January 2020, in final form 23 October 2020)

\begin{abstract}
We present a linear equation for the Walker circulation streamfunction and find its analytic solutions given specified convective heating. In a linear Boussinesq fluid with Rayleigh damping and Newtonian cooling, the streamfunction obeys a Poisson's equation, forced by gradients in the meridionally averaged diabatic heating and Coriolis force. For an idealized convective heating distribution, analytic solutions for the streamfunction can be found through an analogy with electrostatics. We use these solutions to study the response of the Walker circulation strength (mass transport) to changes in the vertical and zonal scales of convective heating. Robust responses are obtained that depend on how the total convective heating of the atmosphere responds to changing scale. If the total heating remains unchanged, increasing the zonal scale or the vertical scale always leads to a weaker circulation. Conversely, if the total heating grows in proportion to the spatial scale, the circulation becomes stronger with increasing scale. These conclusions are shown to be consistent with a threedimensional numerical model. Moreover, they are useful in describing the observed seasonal and interannual (ENSO) variability of the Indo-Pacific Walker circulation. On both time scales, the overturning becomes weaker with increasing zonal scale of the convective region, reminiscent of our solutions where we do not vary the total convective heating. Reanalysis data also indicate that the zonal circulation is quite strongly damped, thus yielding a result that the circulation strength is directly proportional to the warm-pool spatial-mean precipitation.
\end{abstract}

KEYWORDS: Tropics; Heating; Large-scale motions; Walker circulation; Latent heating/cooling; Reanalysis data

\section{Introduction}

Understanding the coupling between convection and largescale atmospheric circulation is key to understanding the tropical climate. Convection, however, is not equally prevalent throughout the tropics, and as a result, large-scale atmospheric overturning circulations develop, with rising branches in the regions of frequent convection, and descending branches in the regions where deep convection is relatively rare. As suggested by Bjerknes (1969), the persistent deep convection over the western Pacific warm pool and radiative cooling over the eastern Pacific lead to a zonally asymmetric atmospheric circulation. Ever since his seminal paper, this circulation has been known as the Walker cell due to its pivotal role in the Southern Oscillation phenomenon of Sir Gilbert Walker.

Many studies have analyzed the response of the tropical atmosphere to spatially localized convective heat sources (e.g., Webster 1972; Gill 1980; Geisler 1981; Hartmann et al. 1984; Wu et al. 2000b; Schumacher et al. 2004; Schubert and McNoldy 2010; Lutsko 2018). However, much less attention has been paid to studying how sensitive the circulation is to changes in the strength and structure of diabatic heating. One of the few studies devoted to the topic is the seminal paper by Hartmann et al. (1984). Using a three-dimensional primitive equation model linearized around a zonal-mean basic state,

๑ Denotes content that is immediately available upon publication as open access.

Corresponding author: Juho Iipponen, iipponen@princeton.edu they examine the effects of varying the convective heating profile from that of unorganized deep convection to a profile more representative of a mature cloud cluster with mesoscale organization. They conclude that the elevated heating profile associated with mature cloud clusters shifts the circulation centers upward, and is able to reproduce the observed westward tilt of the Walker circulation with height. This result was verified by Schumacher et al. (2004), who used observational estimates of convective heating to drive a primitive equation model. However, they find that the tilt in the circulation is primarily driven by zonal gradients in the fraction of stratiform rain over the Pacific, and is not due to an atmospheric wave response as discussed by Hartmann et al. (1984). With an analytic approach, Wu et al. (2000b) similarly compare the deep convective and mature cluster profiles; they conclude that the deep heating profile projects onto vertical modes with greater equivalent depths, hence the circulation response extends farther from the heat source than in the case of the elevated profile.

Using a linear model similar to Hartmann et al. (1984), Geisler (1981) studied the Walker circulation response to changing zonal scale of convective heating. Their prescribed heating used a fixed vertical profile resembling that of deep convection, and the magnitude of the vertically integrated heating was described as a two-dimensional Gaussian distribution centered at the equator. According to their results, circulation to the east of the heat source has larger zonal scale, and is weaker than the circulation to the west of the heating. Narrowing the zonal scale of the heat source from $40^{\circ}$ to $20^{\circ}$ in longitude (and decreasing the total convective heating by the same fraction) leads to weaker circulation, 
and the contrast in strength between the two cells is amplified.

To better describe the changes in the circulation as the shape and magnitude of the heating is varied, we aim to derive an equation relating the zonal streamfunction to the (convective) heating. To our knowledge, such a derivation does not exist in the context of the Walker circulation, but inspiration can be drawn from similar studies on other atmospheric overturning circulations. For a balanced axisymmetric vortex, Eliassen (1951) showed that the meridional streamfunction obeys a generalized Poisson's equation, with source terms due to diabatic heating and frictional torque. Kuo (1956) used a similar set of balanced equations to derive a streamfunction equation for the large-scale meridional overturning circulation, again forced by diabatic heating and friction. This Kuo-Eliassen equation, with some simplifications, can readily be solved analytically; Kuo (1956) and Eliassen (1951) use a Green's function approach to analyze the circulation response to point sources of heat and momentum, and Kuo (1956) presents a closed-form solution for a Hadley circulation forced by a meridional heating gradient.

More recently, Gonzalez and Rojas (2014) solved a simplified form of the Kuo-Eliassen equation, exploring the roles of deep diabatic heating and Ekman pumping in driving the deep and shallow Hadley circulations. Similarly, Schubert and McNoldy (2010) solved the streamfunction equation analytically in the case of a tropical cyclone, for both a diabatic heating resembling the eyewall, and for a uniformly heated ellipse. Both studies explore the effects of static and inertial stability and conclude that large inertial stability constrains the circulation response in the horizontal direction, while large static stability constrains it in the vertical.

In several simplified models of tropical dynamics, however, the horizontal response is instead constrained by linear friction (e.g., Matsuno 1966; Gill 1980; Webster 1972; Zebiak and Cane 1987). These models often require damping time scales on the order of days in order to produce realistic circulations. This rather strong frictional damping has traditionally been attributed to convective momentum transport and nonlinear advection (Holton and Colton 1972; Lin et al. 2008). Recently, however, Stechmann and Ogrosky (2014) used observations of winds and outgoing longwave radiation to conclude that the zonal circulation in the tropics resembles an undamped Kelvin and Rossby wave response. Motivated by this discrepancy, we aim to produce an order-of-magnitude estimate for the damping time scale by comparing our analytic results with the observations.

A common feature of the aforementioned models and the present work is that they analyze the circulation response to prescribed convective heating that is not allowed to couple back to the circulation it induces. However, tropical circulations are largely driven by moist dynamics, whereby convective heating and circulation are tightly coupled (e.g., Emanuel et al. 1994). This two-way coupling is commonly addressed using a moist static energy framework first proposed by Neelin and Held (1987) [see also reviews by Neelin (2007) and Raymond et al. (2009)]. This theory determines the deep circulation as a response to boundary forcing, with the strength of the response governed by the gross moist stability (GMS; a measure of the effective stratification felt by the deep convection). Yu et al. (1998) suggests that the reason the Gill (1980) type models with prescribed heating and constant phase speeds have been successful in studying tropical dynamics is that the GMS is relatively constant in space over the warm pool region. As the GMS governs the propagation of equatorially trapped waves (Neelin and Yu 1994), models with constant static stabilities and fixed vertical profiles of heating yield reasonably realistic circulation structures.

GMS theory solves for the circulation and convective heating given a prescribed external forcing and entropy structure of the atmosphere. In contrast, the models with prescribed convective heating only solve for the circulation. Insofar as the specified heating is consistent with one produced the coupled system, the induced circulation should also resemble the coupled one. Thus, the use of the prescribed heating is not intended to imply there is a one-way connection from heating to circulation. However, ignoring the two-way coupling can be useful for understanding the circulation response in isolation, i.e., ignoring the question of how the heating was formed in the first place.

Among the many parameters that characterize the overturning circulations, we have decided to concentrate on the circulation mass transport (as indicated by the maximum value of the zonal streamfunction). In addition to being a key parameter in its own right, it may also be related to the marine boundary layer cloud cover in the eastern subtropical Pacific. Although the regions of stratocumulus cloud decks are mostly associated with lower sea surface temperatures and elevated inversion strengths (e.g., Klein and Hartmann 1993; Bretherton et al. 2013), large-scale subsidence plays an independent role in controlling the stratocumulus cloud properties. Myers and Norris (2013) showed that, if inversion strength is controlled for, regions of stronger subsidence are associated with reduced boundary layer cloudiness. They also conclude that subsidence is the leading cause of interannual variability in cloud liquid water path, and also contributes significantly to its climatological variability.

The subsidence velocities are directly related to the amount of mass transported by the overturning circulation and to the spatial extent of the subsiding branch. Both are controlled by the zonal structure of convective heating in the equatorial regions, which causes significant variations in the Walker circulation on seasonal (Trenberth et al. 2000; Schwendike et al. 2015), interannual (Bayr et al. 2014; Schwendike et al. 2014) and climatological (e.g., Vecchi et al. 2006; Bayr et al. 2014; Schwendike et al. 2015; He and Soden 2015) time scales. The principal goal of the present study is to develop a simple model for understanding how the zonal circulation responds to changes in the spatial pattern of convective heating.

In this work, we show that the streamfunction equation takes the form of Poisson's equation, with gradients of diabatic heating and Coriolis force as the source terms. Through simple analytic solutions, we explore how the strength of the circulation is affected by a change in the zonal or vertical extent of convective heating. These solutions are then compared with a nonlinear numerical model, showing consistent results. 
Finally, the conclusions drawn from the analytic model are shown to be relevant to describing the seasonal and interannual variability of the Indo-Pacific Walker circulation.

\section{Streamfunction equation}

The streamfunction equation is derived for a Boussinesq fluid, with the equations linearized around a motionless basic state. We use a simplified form of the zonal momentum equation, where the geostrophic balance is modified by Rayleigh damping, and we couple this to a thermodynamic equation with Newtonian cooling (where the advective and diffusive terms have been ignored):

$$
\begin{aligned}
\frac{1}{\rho_{0}} \frac{\partial p}{\partial x} & =-\alpha u+f v, \\
w N^{2} & =J-r B .
\end{aligned}
$$

Here $\rho_{0}$ is a constant reference density, $N^{2}$ is the buoyancy frequency, $J$ is a buoyancy source due to diabatic heating, $r$ is the Newtonian cooling rate, $\alpha$ is the Rayleigh damping rate, and $u, v$, and $w$ are the wind components in the $x, y$, and $z$ directions, respectively. The parameters $\rho_{0}, \alpha, N^{2}$, and $r$ are assumed to be constant, while the Coriolis parameter $f$ can be a function of latitude. Pressure $p$ and buoyancy $B$ are perturbations from a hydrostatic basic state, satisfying

$$
B=\frac{1}{\rho_{0}} \frac{\partial p}{\partial z}
$$

These equations can be combined into one by differentiating Eq. (2) with respect to $x$ and Eq. (1) with respect to $z$, using (3), and setting the two equations equal through the buoyancy gradient terms. After averaging over an arbitrary range of latitudes (denoted with an overbar), the result is

$$
N^{2} \frac{\partial \bar{w}}{\partial x}-\alpha r \frac{\partial \bar{u}}{\partial z}=\frac{\partial \bar{J}}{\partial x}-r \frac{\partial \overline{f v}}{\partial z} .
$$

In this work we only consider the divergent wind in the definition of the streamfunction (e.g., Schwendike et al. 2014), and do not solve for the rotational wind that plays no part in the circulation mass transport. This way, the horizontal derivatives of the streamfunction correspond to the vertical velocities driven by the divergence of the zonal wind on the zonal plane. We thus define a streamfunction $(\psi)$ for the meridionally averaged circulation as a vertical integral of the divergent zonal wind $\left(\bar{u}_{\text {div }}=\bar{u}-\bar{u}_{\text {rot }}=-\partial \psi / \partial z\right.$, where $\bar{u}_{\text {rot }}$ is the rotational wind component; $\bar{w}=\partial \psi / \partial x)$. With this definition, the streamfunction equation becomes

$$
\left(\frac{\partial^{2}}{\partial x^{2}}+\Lambda \frac{\partial^{2}}{\partial z^{2}}\right) \psi=\frac{1}{N^{2}} \frac{\partial \bar{J}}{\partial x}-\frac{r}{N^{2}} \frac{\partial \overline{f v}}{\partial z}+\frac{\alpha r}{N^{2}} \frac{\partial \bar{u}_{\mathrm{rot}}}{\partial z},
$$

where $\Lambda=r \alpha / N^{2}$ is a scale parameter.

The main way our derivation differs from the derivation of the streamfunction equation for the meridional overturning circulation is in the way the thermal and momentum equations are coupled. These Kuo-Eliassen type equations (e.g., Kuo 1956; Schubert and McNoldy 2010; Gonzalez and Rojas 2014) assume geostrophic (or gradient) wind balance between the zonal wind and the meridional pressure gradient. However, because of the smallness of the Coriolis parameter in the deep tropics, the meridional wind is not in a geostrophic balance with the zonal pressure gradients. Thus, such a balance condition cannot easily be used in the derivation of the Walker streamfunction equation. For our system, the vertical coupling instead occurs through the Newtonian cooling term in (2) - in contrast to the Kuo-Eliassen equation, which is typically derived for an inviscid flow. The derivation of our streamfunction equation also requires the use of a meridionally varying $f$, which leads to the emergence of the rotational (covariance) term on the right-hand side of (5) (there is no analogy in the Kuo-Eliassen equation, since $f$ does not vary when the equations are zonally averaged).

Despite stark differences in the derivations, Eq. (5) strongly resembles the Kuo-Eliassen equation in a case with no baroclinicity and weak horizontal flow [e.g., Gonzalez and Rojas 2014, Eq. (10)]. In both cases, the flow is being driven by horizontal gradients in diabatic heating. There are also similarities in the interpretation of the scale parameter $[\Lambda$ in Eq. (5)]. The ratio of the inertial to static stability is a key parameter in the Kuo-Eliassen equation, as it determines the scale factor of the circulation response (Schubert and McNoldy 2010). In our $\Lambda$, however, the role of the inertial stability is played by the Rayleigh damping. For high Rayleigh damping rates (analogous to high inertial stability), the disturbances decay away quickly in the zonal direction, and the circulation has a shorter zonal scale. The vertical scale, in turn, is determined by both the Newtonian cooling and the static stability. Newtonian cooling couples the vertical levels together, reducing vertical wind shear, and increasing the vertical extent of the response (Wu et al. 2000a). This effect can be further amplified by weak static stability, requiring air parcels to move at greater vertical velocities (and thus have greater vertical displacements) to balance the heating.

\section{Analytic solutions}

For simplicity, the analytic solutions we present in this work concern the circulation in the absence of rotation:

$$
\left(\frac{\partial^{2}}{\partial x^{2}}+\Lambda \frac{\partial^{2}}{\partial z^{2}}\right) \psi=\frac{1}{N^{2}} \frac{\partial \bar{J}}{\partial x}
$$

Inclusion of rotation would require a parameterization or simplified modeling of the complete zonal and meridional velocities. This would add complexity in the solutions that may not be necessary for the qualitative understanding of circulation behavior. We thus begin by deriving the nonrotating solutions, but will return to the question of rotational effects at the end of the section by drawing a comparison with the Gill (1980) model.

In a manner very similar to Tarshish et al. (2018), we note that Eq. (6) has an analogy in the field of electrostatics. According to Gauss's law, the electric field (E) created by a charge distribution $Q_{C}$ is 

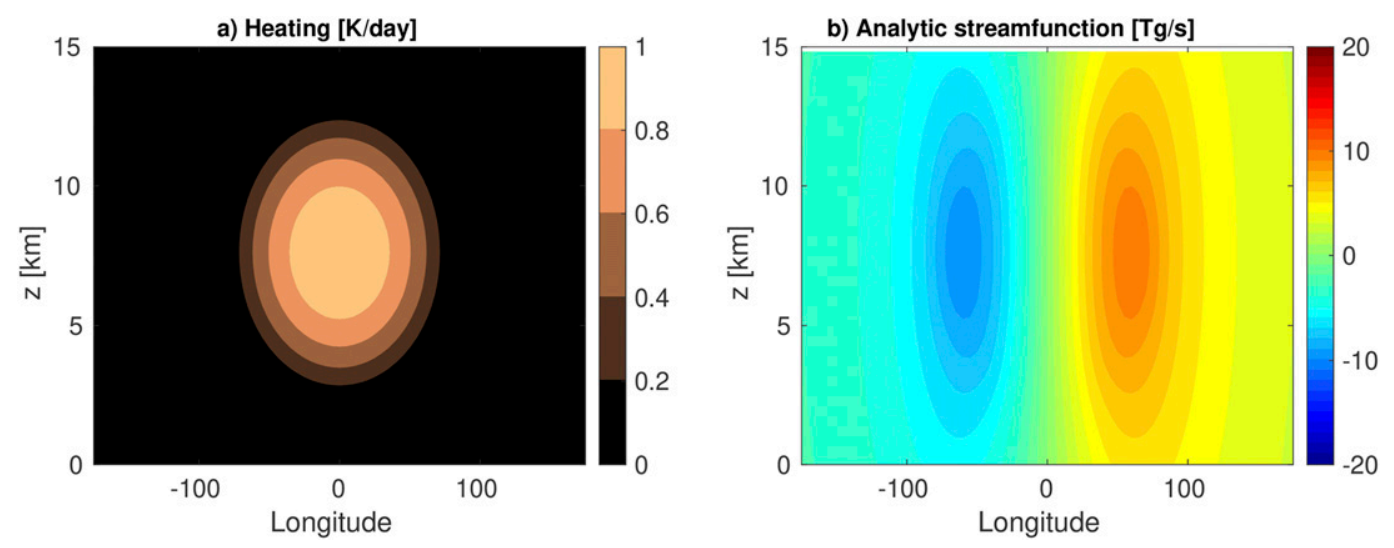

FIG. 1. (a) Illustration of a meridionally averaged heating profile of Eq. (10) with an amplitude of $1 \mathrm{~K} \mathrm{day}^{-1}$, $H^{z}=5 \mathrm{~km}$, and $L=80^{\circ}$. (b) The free-space analytic streamfunction solution for heating in (a) using $\Lambda=3.6 \times 10^{-6}$.

$$
\nabla_{x z} \cdot \mathbf{E}=\frac{Q_{c}}{\varepsilon_{0}}
$$

where $\nabla_{x z}$ is the $2 \mathrm{D}$ gradient operator in $x$ and $z$ directions, and $\varepsilon_{0}$ is the vacuum permittivity. Differentiating Eq. (7) with respect to $x$, and expressing the zonal charge distribution gradient as a Laplacian of a scalar function $\psi$ :

$$
\nabla_{x z} \cdot \frac{\partial \mathbf{E}}{\partial x}=\frac{1}{\varepsilon_{0}} \frac{\partial Q_{c}}{\partial x}=\nabla_{x z}^{2} \psi .
$$

Equation (8) resembles the nonrotating streamfunction Eq. (6), when the scale parameter is absorbed into a scaled vertical coordinate: $Z=z / \sqrt{\Lambda}$. Thus, in free space, far away from boundaries,

$$
E^{x}=\psi
$$

The streamfunction $(\psi)$ due to a given heat distribution is hence analogous to the $x$ component of the electric field $\left(E^{x}\right)$ produced by a charge distribution of the same shape.

Therefore, solutions derived in the electrostatics literature can readily be used to solve for the streamfunction, given some prescribed heating (charge density) distribution, and the nonrotating form of Eq. (5): Furman (1994) presents solutions for the two-dimensional electric fields produced by cylindrically symmetric, elliptical charge distributions. The simplest shape that qualitatively describes the observed vertical and zonal structure of diabatic heating is that of a parabola [see, e.g., Tao et al. (2016) for a review]:

$$
\bar{J}=\left\{\begin{array}{cl}
\overline{J_{0}}\left(1-\frac{x^{2}}{L^{2}}-\frac{Z^{2}}{H^{2}}\right), & \frac{x^{2}}{L^{2}}+\frac{Z^{2}}{H^{2}} \leq 1 \\
0, & \frac{x^{2}}{L^{2}}+\frac{Z^{2}}{H^{2}}>1
\end{array},\right.
$$

where $J_{0}$ is the amplitude of the buoyancy source $\left(\mathrm{m} \mathrm{s}^{-3}\right), L$ is the zonal and $H$ is the vertical scale of the heating (scaled from true geometric height $H^{z}$ as $H=H^{z} / \sqrt{\Lambda}$ ), and $Z$ is a similarly scaled vertical coordinate. This heating pattern is illustrated in Fig. 1a for $L=80^{\circ}$ longitude, $H^{z}=5 \mathrm{~km}$, and an $T_{0} J_{0} / g=1 \mathrm{~K}$ day $^{-1}$ (with $g$ the acceleration due to gravity and
$T_{0} 250 \mathrm{~K}$ a constant reference temperature). Given a parabolic distribution, the streamfunction in the interior of the heat source is (Furman 1994)

$$
\begin{aligned}
\psi_{\text {interior }}= & \operatorname{Re}\left\{\frac{\overline{J_{0}} L H\left(\frac{x}{L}+\frac{i Z}{H}\right)}{N^{2}(L+H)}\right. \\
& \left.\times\left(1-\frac{\left[2(x-i Z)+\frac{H x}{L}-\frac{i L Z}{H}\right]\left(\frac{x}{L}+\frac{i Z}{H}\right)}{3(H+L)}\right)\right\} .
\end{aligned}
$$

An analytic solution also exists for the exterior, but is not required in the derivation of the results presented in this work. For completeness, the full streamfunction solution is shown in Fig. $1 \mathrm{~b}$ (with $\rho_{0}=0.67 \mathrm{~kg} \mathrm{~m}^{-3}, r^{-1}=\alpha^{-1}=12 \mathrm{~h}, N^{2}=1.5 \times$ $\left.10^{-4} \mathrm{~s}^{-2}\right)$. Since the circulation response is decaying away from the heating boundary, and because of symmetry, the maximum value of the streamfunction is at the level of maximum heating (inside the heated region). We thus find the total mass transport $M$ (streamfunction maximum in units of $\mathrm{kg} \mathrm{s}^{-1}$ ) as

$$
M=\frac{4}{3 \pi} \frac{g \int_{V} Q d m}{c_{p} T_{0} N^{2} \Lambda^{1 / 2}} \sqrt{\frac{1}{2 L^{2}+3 L H+H^{2}}},
$$

where $\int_{V} Q d m \equiv Q_{\mathrm{tot}}=c_{p} T_{0} \overline{J_{0}} \rho_{0} \Delta y H^{z} L \pi / 2 g$ is the atmospheric diabatic heating $\left(Q\right.$, in units of $\left.\mathrm{W} \mathrm{kg}^{-1}\right)$ integrated over the mass $(m)$ of the meridional channel $(V)$ with a width of $\Delta y$. Equation (12) is the main result of this work, and our next goal is to use it to understand how the mass transport depends on the scale parameters $H$ and $L$.

It is not obvious, however, as to how the total heating $\left(Q_{\text {tot }}\right)$ should behave as a function of scale. Here, we use two different approaches, which are illustrated in Fig. 2. In the first approach (hereafter referred to as the domain-proportional case; thin solid line in Fig. 2), the increase in total heating is directly proportional to increasing vertical or horizontal scale. This is equivalent to 


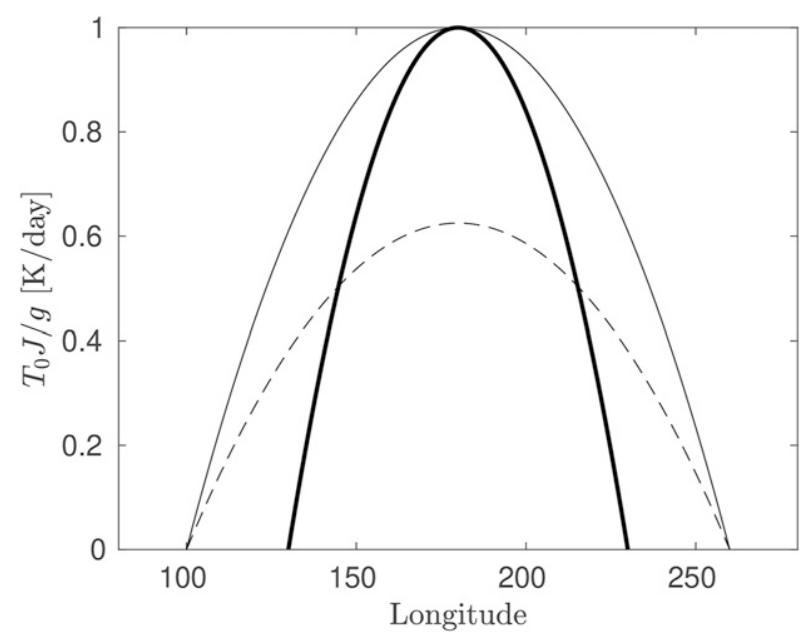

FIG. 2. An example of the meridionally averaged convective heating (at the level of maximum heating) for a convective region with a width of $100^{\circ}$ (thick solid line). The behavior of the heating pattern, as the convective region widens to $160^{\circ}$, is shown for both the domain-proportional (thin solid line) and the constant total heating (thin dashed line) case. The thick solid and dashed curves enclose an equal area, while the two solid lines have the same mean; these are general features of the constant total and the domain-proportional experiments, respectively.

holding the heating amplitude $J_{0}$ constant. In the second approach (hereafter referred to as the constant total heating case; dashed line in Fig. 2), we simply keep $Q_{\text {tot }}$ constant. This might occur if the total convective heating is constrained by atmospheric energetics (radiative cooling). It should be noted, however, that there can be competing effects of changing the heating gradient and changing the total amount of heating. In the domain-proportional approach, increasing (decreasing) the horizontal scale of the heating increases (decreases) $\int_{V} Q d m$, but in turn decreases (increases) the magnitude of the heating gradient $(\partial \bar{J} / \partial x)$. The constant total heating experiments thus help us to understand these competing effects by only considering the gradient effect.

Figure 3 shows, for both cases, the analytic solutions for the streamfunction maximum as a function of the zonal and vertical scales (with $T_{0} J_{0} / g=3 \mathrm{~K}_{\text {day }}{ }^{-1}$ at $\left(L, H^{z}\right)=(5600 \mathrm{~km}$, $2.5 \mathrm{~km})$ ). Two different values for the scale parameter $\Lambda$ have been used, one with $r^{-1}=\alpha^{-1}=5$ days $\left(\Lambda=3.6 \times 10^{-8}\right)$ and the other with $r^{-1}=\alpha^{-1}=12 \mathrm{~h}\left(\Lambda=3.6 \times 10^{-6}\right)$. For both values of the scale parameter, the circulation response very much depends on the behavior of the total heating as a function of scale, leading to changes of opposite signs in the two cases. Increasing scale always leads to a stronger circulation in the domain-proportional case, but has the exact opposite effect in the constant total heating case. Thus, according to the domainproportional solution, the effect of increasing the total heating with scale always overpowers the opposing effect of the weaker heating gradient. The effect of the scale parameter changes the circulation sensitivity and strength. Strong circulation is associated with weak damping if atmospheric stability is held constant.

In the domain-proportional case, the sensitivity to scale depends mostly on the aspect ratio of the heating distribution.
If $H>L(H<L)$, the circulation responds more strongly to the horizontal (vertical) scale, and for $H \gg L(H \ll L)$, the response becomes completely insensitive to $H(L)$. In the constant total heating case, the circulation is strong and sensitive to scale at the smaller scales, but becomes weaker and less sensitive as the scales grow larger. Stronger damping (larger $\Lambda$ ) causes the circulation to respond mostly to $L$ in the constant total heating case, but has the effect of reducing this sensitivity in the domain-proportional case (for the same value of $H^{z}$ ).

Some insight into the robustness of these results, and to the effects of rotation can be gained by examining the Gill (1980) solutions in a similar setting. The zonal momentum and thermodynamic equations in the Gill model use the same simplifications as our Eqs. (1) and (2), except for the vertical structure, which is assumed to be that of the gravest baroclinic mode with a constant equivalent depth. The model also makes use of a meridional momentum equation that has the form of a geostrophic balance. Given a pattern of heating that is described by a sinusoidal in zonal direction and a low-order parabolic cylinder function in the meridional direction, analytic solutions for the stationary Kelvin and long planetary wave modes can be found. In the Gill (1980) model, the zonal overturning circulation is a combined response of these two wave types to heating, with the Kelvin waves propagating to the east of the heat source and the planetary Rossby waves propagating to the west.

To analyze if this wave response is consistent with our nonrotating model, we use the formulae provided by Gill (1980) in his Eq. (4.17), numerically solving for the zonal mass transport in both cells for a variable zonal heating scale $L$. Figure 4 shows the sensitivity of the transport in the two cells as a function of zonal scale of heating, for both the domainproportional and the constant total heating experiments. In general, increasing scale in the domain-proportional case leads to a stronger circulation, while the opposite is true if the total heating is held constant. The western cell, however, is consistently weaker than the eastern cell, and has a different sensitivity to zonal scale. This asymmetry could potentially be included in our model through the rotational term [last term on the right-hand side of Eq. (5)]. According to the Gill solutions, on the east side of the heating the meridional wind vanishes due to the Kelvin wave response, while on the western side the lower level wind is equatorward due to planetary Rossby waves (cf. Gill 1980, Fig. 1). Thus, most of the rotational effects occur in the west, where the two forcing terms partly offset one another, possibly explaining the weaker circulation there. Additionally, in the constant total heating experiment, the strength of the western circulation falls more rapidly with increasing scale. Likewise, in the domain-proportional case, the strength becomes insensitive to $\mathrm{L}$ at shorter scales in the west than in the east, with the exception of slight weakening in the former for large scales (an effect not captured by our nonrotating solutions). These patterns hold true independent of the value of damping used (not shown). The changes in sensitivity and strength are hence quite consistent with our nonrotating solutions, in which the transport in the western cell resembles a circulation with stronger damping. However, the fact that the transports are similar does not mean that the 


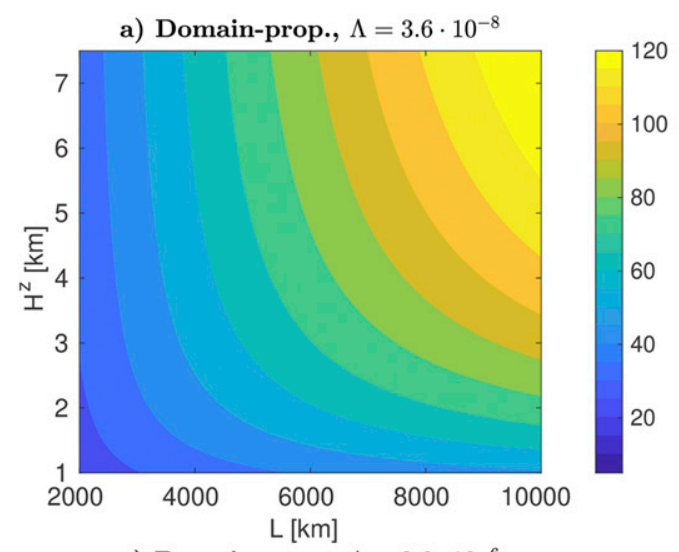

c) Domain-prop., $\Lambda=3.6 \cdot 10^{-6}$

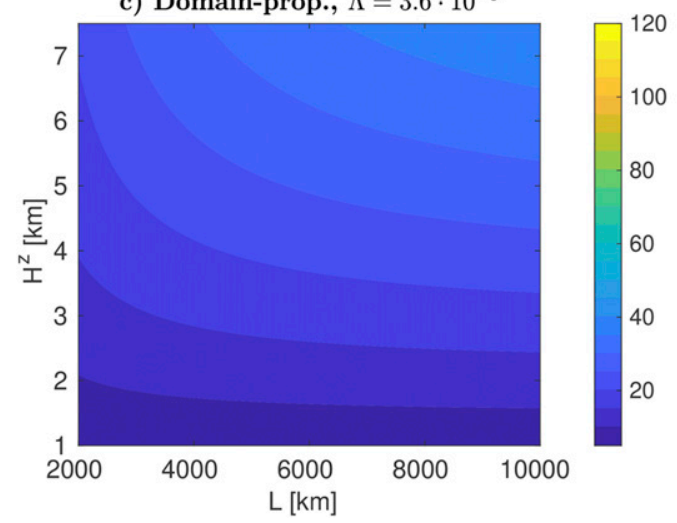

b) Constant total, $\Lambda=3.6 \cdot 10^{-8}$

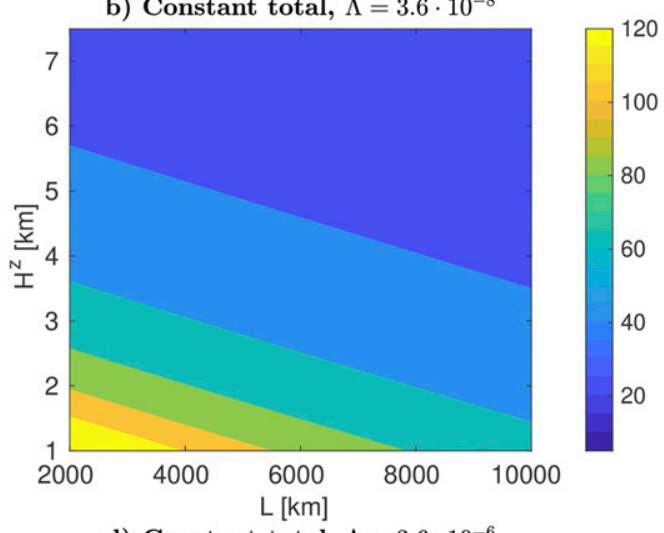

d) Constant total, $\Lambda=3.6 \cdot 10^{-6}$

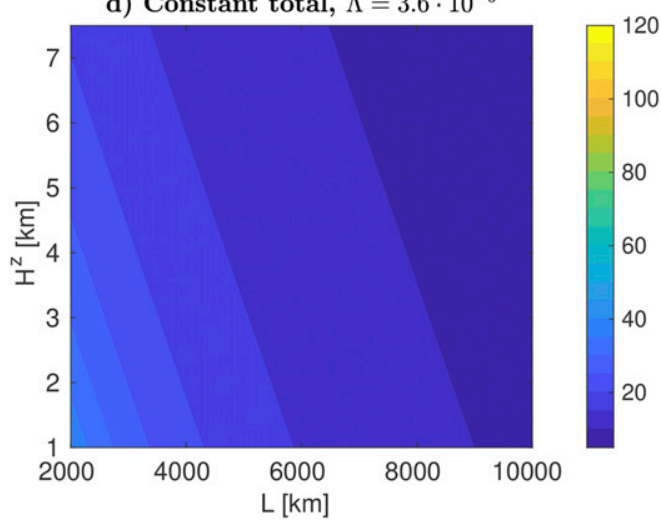

FIG. 3. Overturning circulation mass transport $M\left(\mathrm{Tg} / \mathrm{s}\right.$ or $\left.10^{9} \mathrm{~kg} \mathrm{~s}^{-1}\right)$, in a domain with a width of $40^{\circ}$ latitude) as a function of zonal $(L)$ and vertical $\left(H^{z}\right)$ scales for (a) the domain-proportional and (b) the constant total heating case with weak damping $\left(\Lambda=3.6 \times 10^{-8}\right)$. (c), (d) As in (a) and (b), but for strong damping $\left(\Lambda=3.6 \times 10^{-6}\right)$.

complete two-dimensional structure of the streamfunction would be unaffected by the inclusion of rotation; Gill's (1980) Fig. 1c shows considerable differences in the streamfunction between the two sides. Furthermore, a systematic difference in strength between the two cells is not observed in our numerical results or in reanalysis (see the discussion in sections 4 and 5).

\section{Numerical models}

The analytic solutions are verified in a fully nonlinear numerical model. In addition to including the effects of boundary conditions (ground and stratosphere), this model includes nonlinear advection terms, and a meridional temperature gradient. The effect of rotation is analyzed by running the model with the angular velocity of the planet set to an Earthlike value $\left(7.29 \times 10^{-5} \mathrm{~s}^{-1}\right)$ or simply set to zero. We use the Held and Suarez (1994) forcing, which includes spatially nonuniform Newtonian cooling and Rayleigh damping rates, with maximum values in the planetary boundary layer. To produce realistic static stabilities, however, the Newtonian cooling time scale in the free troposphere must be decreased from 40 to 8 days, and a moist convective parameterization must be used. We employ a Betts-Miller-type scheme as described in Frierson (2007), and assume a fully saturated atmosphere. We run the simulation at $\mathrm{T} 42$ resolution for 25000 days, with the first 2000 days excluded.

The model is forced with heating distributions, whose meridional structure is parabolic and whose meridional means are of the form given by Eq. (10). Three different shapes are used to analyze the response:

- Narrow stratiform heating distribution (the narrow-stratiform case), with a zonal width $\left[=2 L\right.$ in Eq. (10)] of $100^{\circ}$, and a vertical extent from 650 to $150 \mathrm{hPa}$.

- Narrow deep convective distribution (the narrow-deep case) with the same width and top as the narrow-stratiform case, but with a base at $850 \mathrm{hPa}$.

- Wide deep convective distribution (the wide-deep case), with the same vertical extent as in the narrow-deep case, but with a zonal width of $160^{\circ}$.

We run the model for both the constant total and the domainproportional cases. The zonal mean of the prescribed heating is assumed to drive the Hadley circulation (e.g., Geisler 1981), while the positive anomaly from the zonal mean ( $Q^{*}$ : the gray region in Fig. 5a) acts as the driver for the Walker circulation. In the constant total heating experiment, the magnitude of this positive anomaly is kept constant (1.5 PW), while in the domain-proportional runs its magnitude is directly proportional to horizontal and vertical scales. In all experiments, 


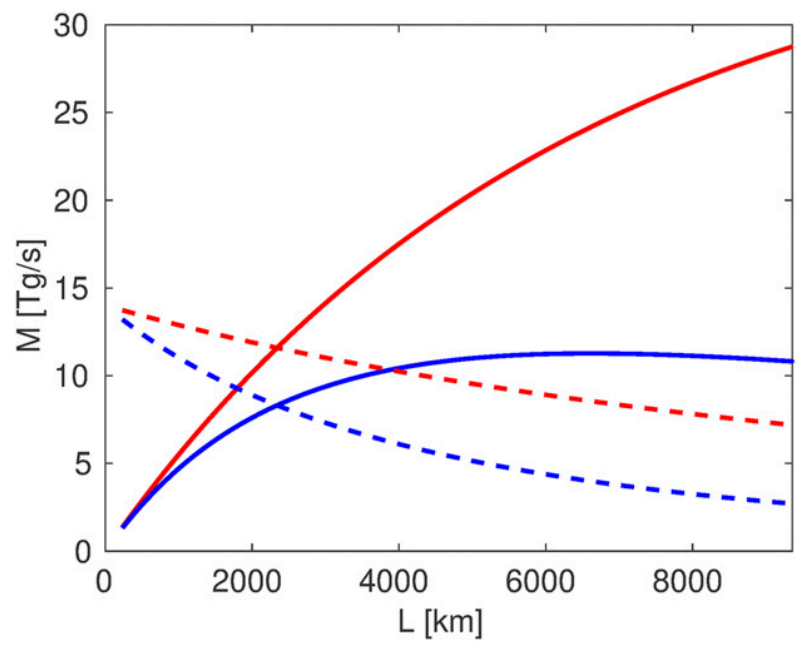

FIG. 4. Behavior of the Walker circulation mass transport in the Gill (1980) model on the eastern side (red) and western (blue) side of the heated region as a function of (nondimensional) horizontal scale of heating $L$ for the domain-proportional (solid) and constant total heating (dashed) cases. See text and Gill (1980) for details.

we keep the meridional width of the heating constant at $40^{\circ}$ latitude $\left(20^{\circ} \mathrm{S}\right.$ to $\left.20^{\circ} \mathrm{N}\right)$, centered at the equator.

Figure 5 shows the vertically integrated prescribed heating from the nonlinear model and the associated streamfunction in the domain-proportional wide-deep case. The meridional averages are taken over the width of the heating region, and divergent winds are used for computing the streamfunctions (as in Schwendike et al. 2014). The streamfunction is qualitatively very similar to the analytic solution (cf. Fig. 1) in terms of shape, but has some asymmetry between the western and eastern cells-most likely caused by different wave characteristics on the two sides (e.g., Gill 1980). The observed westward tilt with height of the eastern cell is very likely the result of Kelvin waves emanating from the heated region with a downward group velocity (e.g., Hartmann et al. 1984). Whether this explains the fact that the streamfunction maximum is at a lower altitude in the east is unclear, and the answer probably lies in the interaction of this flow structure with the convective parameterization.

Table 1 summarizes the results from the model. It shows the response of the Walker circulation strength to changes in the heating distribution, for both the constant total and the domain-proportional cases, in both the rotating and nonrotating configurations. For comparison with the numerical model, the analytic results [Eq. (12)] for the same heating distribution with strong $\left(\Lambda=3.6 \times 10^{-6}\right)$ and weak $\left(\Lambda=3.6 \times 10^{-8}\right)$ damping are also shown. It is noticeable that that the sign of the sensitivity is consistent in all models; an increasing scale always leads to a stronger (weaker) circulation in the domain-proportional (constant total) heating case. Rotation, boundary conditions and nonlinear effects would thus appear not to distort the qualitative insights provided by the analytic solutions.

The rotating nonlinear model, however, shows east to west asymmetries in both the strength and the sensitivity of the circulation, which are absent in the nonrotating solutions. Although not the key finding of this study, it is noteworthy that one cell is not consistently stronger than the other, as it appears to be in, e.g., Geisler (1981) and Gill (1980). The nonrotating solutions are stronger due to lower tropical surface temperature and static stability (not shown), but still yield sensitivities consistent in sign with the rotating experiments. It is unclear as to what degree the differences between the two configurations are attributable to differences in the mean state, or the simplified model physics. However, comparing the sensitivities of the western and eastern cells to the strongly and weakly damped analytic solutions reveals a pattern similar to what we observed in the Gill solutions (cf. section 3). Although the weakly damped solutions are much stronger than the cells in the rotating numerical model, their sensitivity somewhat resembles that of the eastern cell [stronger sensitivity to horizontal (vertical) change in the domain-proportional (constant total heating) case]. In a similar way, the western cell behaves more like the strongly damped solution. Interestingly, though, the nonrotating cell resembles the weakly damped solutions
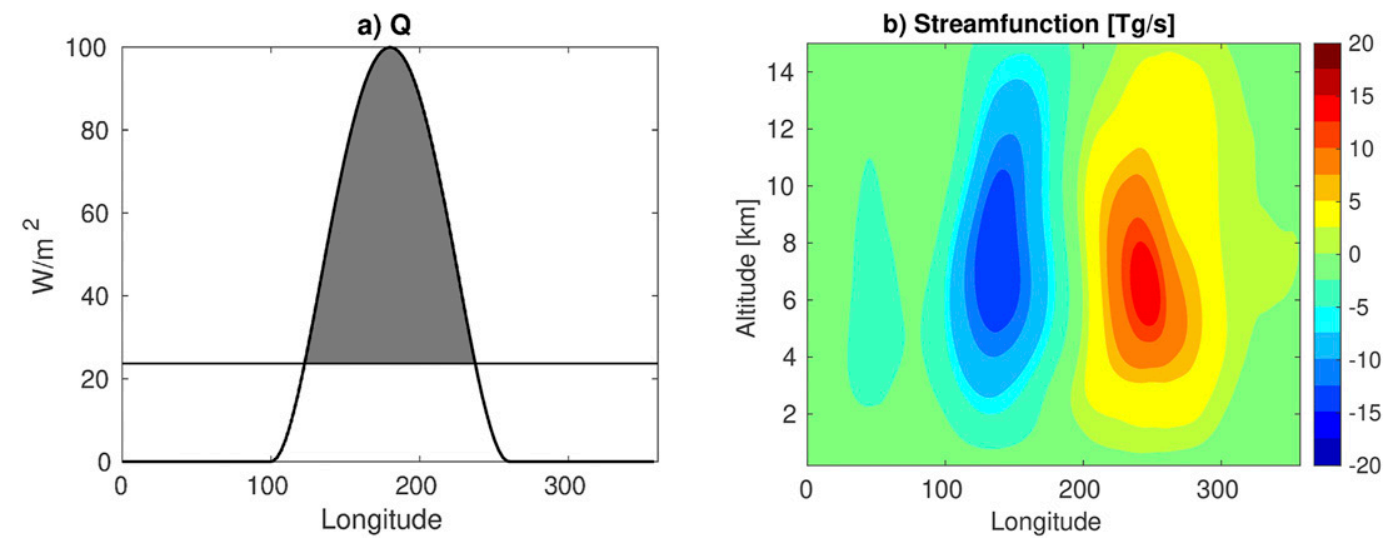

FIG. 5. (a) Meridionally averaged prescribed heating in the domain-proportional wide-deep experiment: shaded region represents the perturbation $\left(Q^{*}=3.3 \mathrm{PW}\right)$ from the zonal mean (black horizontal line). (b) Streamfunction given by the nonlinear model. Averages are computed over the heated region $\left(20^{\circ} \mathrm{S}-20^{\circ} \mathrm{N}\right)$. See text for details. 
TABLE 1. Nonlinear model results. The shape and magnitude of the zonal heating anomaly $\left(Q^{*} ;\right.$ units: PW) is varied, leading to changes in the zonal circulation mass transport $\left(M^{W}\right.$ in the west and $M^{E}$ in the east for the rotating case; $M^{\mathrm{NR}}$ in the nonrotating case; $M^{\mathrm{ASD}}$ and $M^{\mathrm{AWD}}$ for the analytic solutions with $\Lambda=3.6 \times 10^{-6}$ and $\Lambda=3.6 \times 10^{-8}$, respectively; units: $10^{9} \mathrm{~kg} \mathrm{~s}^{-1}$ ). The rightmost column shows the relative changes in strength as either the zonal scale or the vertical scale of the heating is varied.

\begin{tabular}{lccc}
\hline \hline & $Q^{*}$ & $-M^{W}, M^{E}, M^{\mathrm{NR}}, M^{\mathrm{ASD}}, M^{\mathrm{AWD}}$ & $\Delta M^{W}, \Delta M^{E}, \Delta M^{\mathrm{NR}}, \Delta M^{\mathrm{ASD}}, \Delta M^{\mathrm{AWD}}$ \\
\hline $\begin{array}{l}\text { Domain-proportional heating } \\
\quad\end{array}$ & & & \\
$\quad$ Narrow stratiform & 1.5 & $10.0,9.9,39.3,8.5,29.9$ &,,,,----- \\
$\quad$ Narrow deep & 2.0 & $12.5,11.3,44.1,11.0,32.6$ & $+21 \%,+15 \%,+12 \%,+29 \%,+9 \%$ \\
$\quad$ Wide deep & 3.3 & $12.8,14.1,68.2,12.2,46.0$ & $+2 \%,+28 \%,+55 \%,+11 \%,+41 \%$ \\
Constant total heating & & & $-17 \%,-27 \%,-24 \%,-8 \%,-22 \%$ \\
$\quad$ Narrow deep & 1.5 & $8.6,7.2,29.9,7.8,23.3$ & $-37 \%,-8 \%,-29 \%,-30 \%,-12 \%$ \\
Wide deep & 1.5 & $5.4,6.6,21.1,5.5,20.5$ & \\
\hline
\end{tabular}

in the domain-proportional case, but not in the constant total heating case.

\section{ERA5}

We use ERA5 data (Copernicus Climate Change Service 2017) to investigate whether the theoretical results can explain the observed variability in the Walker circulation. The interannual variability is illustrated in Fig. 6, which shows the zonal streamfunctions for El Niño and La Niña together with the meridionally averaged precipitation. The El Niño (La Niña) composite includes all months with the Niño 3.4 index anomaly (from the 1981 to 2010 mean) greater than (less than) $1^{\circ} \mathrm{C}$. Bayr et al. (2014) employ similar methodology, and derive streamfunctions, which strongly resemble those in Fig. 6.

In the light of our theoretical results, we study how the strength of the zonal overturning responds to changes in atmospheric heating. Analogously to the numerical simulations, we define the heating as the positive precipitation anomaly from the zonal mean (gray shading in Fig. 6). Although the two positive anomalies (shaded) during El Niño are separated by a narrow region of below-mean precipitation over the Maritime Continent, we consider them as a single region due to there being very little subsidence between them. During the transition from El Niño to La Niña, the circulation grows stronger on both the eastern ( $+55 \%$ from 9.8 to $\left.15.1 \times 10^{9} \mathrm{~kg} \mathrm{~s}^{-1}\right)$ and the western $\left(+56 \%\right.$ from -11.6 to $\left.-18.1 \times 10^{9} \mathrm{~kg} \mathrm{~s}^{-1}\right)$ side of the convective region. Associated with this change is a significant $(62 \%)$ increase in spatial-mean precipitation anomaly $(P)$, and a reduction in the width of the convective region (from $164^{\circ}$ to $118^{\circ}$ longitude). This increase in circulation strength with decreasing width is consistent with our constant total heating experiments. Furthermore, the relative increase in $P$ is very similar in magnitude to the change in the circulation strength, with the change in the total anomalous precipitation (17\%) being much smaller.

Next, we analyze, whether the circulation changes seen on the interannual scales are applicable on the seasonal time scales, as well. Figure 7 shows the Walker circulation strength as a function of spatial-mean precipitation and (a reciprocal of) the zonal width of the convective region. The eastern cell displays a significant positive correlation between the heating power and circulation strength. Like in the case of interannual variability, the circulation grows stronger when the zonal scale of the heating region is reduced, again consistent with the constant total heating paradigm. The western cell follows a similar pattern for most of the seasonal cycle. However, during the first 3 months of the year, when the Indian Ocean and southern Asia receive little rainfall (the Asian winter monsoon), the constant total heating relationship seems to break down. During this time, there is some uncertainty in the definition of the convective heating region, given that the precipitation anomalies (from the zonal mean) are very small (not shown). It is thus possible that our simple method of estimating the convective heating is not accurate enough during the dry monsoon, but investigation into the cause of the discrepancy is beyond the scope of this study. It is noteworthy, however, that during these months (and in December), the circulation in the east is stronger than it is in the west.

The strong linearity observed in both cells suggests looking for a relationship between the spatial-mean precipitation and circulation strength. To help guide our derivation, we compare the ERA5 circulation observations to the analytic solutions [Eq. (12)] driven by ERA5 precipitation. The total heating $\left(\int_{V} Q d m\right)$ and zonal scale are again analyzed using the precipitation anomalies, and a reasonable vertical scale $\left(H^{z}\right)$ of $5 \mathrm{~km}$ is assumed. The major unknown, however, is the value of the scale parameter $\Lambda$, the uncertainty in which mainly comes from the damping terms. With the value of the static stability held as constant (cf. section 2), we vary the strength of the Newtonian cooling and Rayleigh damping, and observe how their values impact the root-mean-square error (RMSE) between the ERA5 observations and the analytic solutions. Figure 8a shows a comparison between the ERA5-forced analytic solutions and the observed circulation in the east with relatively strong damping $\left(\sqrt{r^{-1} \alpha^{-1}}=2\right.$ days). In Fig. 8b, the RMSE between the analytic solutions and observations is plotted against the damping time scale $\left(\sqrt{r^{-1} \alpha^{-1}}\right)$ for both the eastern and the western (ignoring the Asian winter monsoon) cells. For both sides, large errors are seen with both very short and very long damping time scales, with the smallest error at around 2 to 4 days. In contrast to the Gill model and our numerical results, the reanalysis would indicate that the western cell experiences weaker damping. This is largely a result of the western branch being stronger for most of the year (cf. Fig. 7). The fact that the western cell is, though, not consistently stronger in our numerical model or in ERA5 may indicate that nonlinear effects or details of the atmospheric stability and 


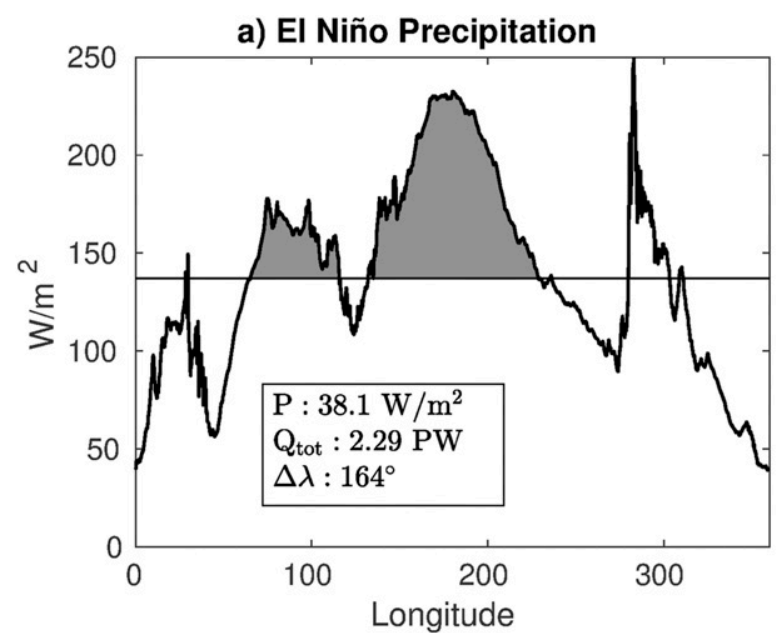

$-20$ $-10$

0

10

20

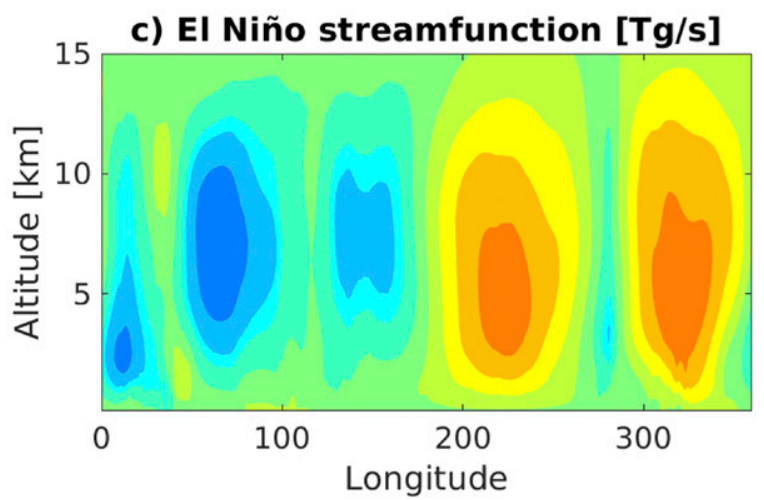

b) La Niña Precipitation

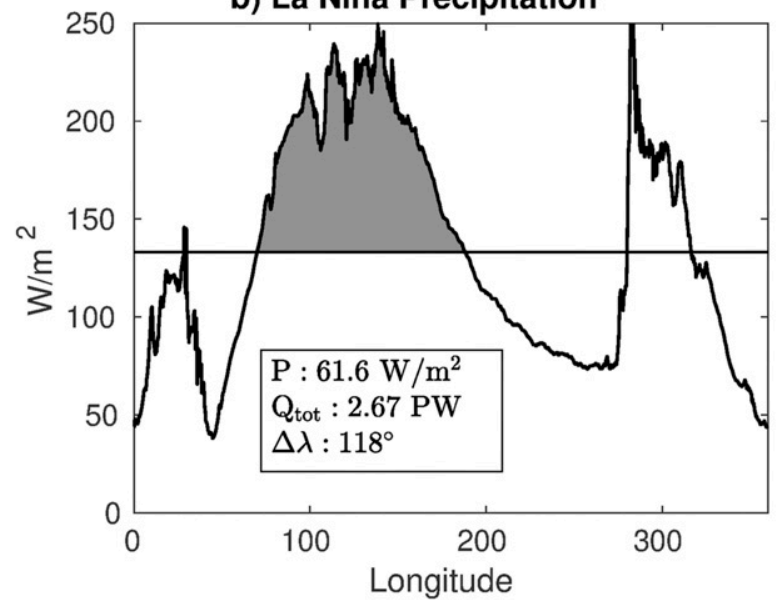

$-20$

$-10$

0

10

20

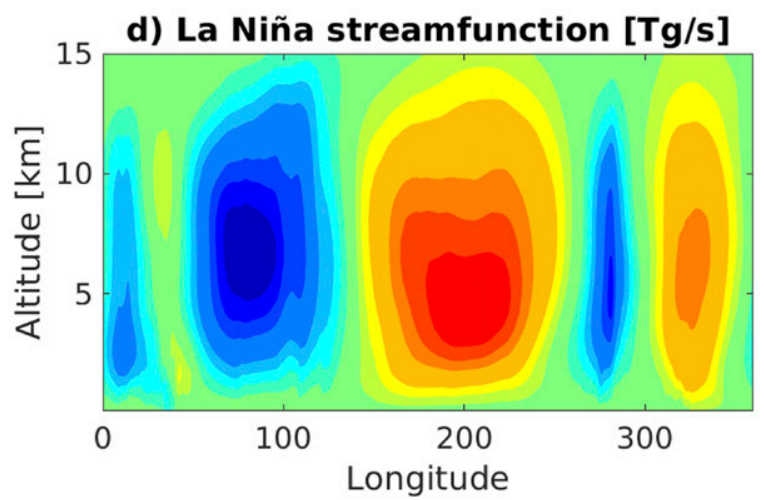

FIG. 6. (top) ERA5 precipitation (1979-2018) for (a) El Niño and (b) La Niña, averaged from $15^{\circ} \mathrm{S}$ to $15^{\circ} \mathrm{N}$. Gray shaded areas indicate the positive anomaly of latent heating from the zonal mean (thin horizontal line). The spatial-mean precipitation anomaly $(P)$, total heating anomaly $\left(\mathrm{Q}_{\text {tot }}\right)$, and zonal width $(\Delta \lambda)$ of these regions are shown in the text boxes. (bottom) Walker circulation streamfunctions from ERA5 divergent zonal wind for (c) El Niño and (d) La Niña, averaged from $15^{\circ} \mathrm{S}$ to $15^{\circ} \mathrm{N}$; contour interval is $2.5 \times 10^{9} \mathrm{~kg} \mathrm{~s}{ }^{-1}$.

convective heating play a role in determining the side on which the mass transport is greater. However, because of the numerous uncertainties, the difference in time scales should not be used to conclude which one of the cells experiences stronger damping.

The most significant simplifications in our analysis are the constant parabolic shape of the convective heating and the computation of the heating based on the precipitation anomaly. There is particular sensitivity to the magnitude of the heating, and the damping time scales increase quickly as the total heating is reduced (not shown); scaling down $Q_{\text {tot }}$ (artificially) by a factor of 2 would push the time scale to 10 days and beyond. The actual diabatic heating rates are very difficult to obtain from the reanalysis data, and the total rates also include contributions from advection, eddies, and radiation. Hence, the results should not be regarded as accurate quantitative estimates, and they should be interpreted strictly in the context of our model. However, they indicate that the very short (less than order a day) or long (greater than order 10 days) time scales are improbable in the light of our model, should the precipitation give accurate estimates of the total diabatic heating. Our analytic solution is then in line with other simple models (e.g., Matsuno 1966; Gill 1980; Webster 1972; Zebiak and Cane 1987) in that relatively strong damping (on the order of several days) is required to obtain a realistic circulation.

With these caveats in mind, we take the strong-damping limit of the analytic solution [Eq. (12)]:

$$
\lim _{r, \alpha \rightarrow \infty} M \propto \lim _{L \gg H} M=\frac{4}{3 \pi} \frac{g}{c_{p} T_{0} N^{2} \Lambda^{1 / 2}} \frac{\int_{V} Q d m}{L} \propto P .
$$

As the seasonal variability of static stability in the observations is small (not shown), the limit indicates the strength of the overturning is linearly proportional to spatial-mean precipitation anomaly $(P)$. This can explain their strong correlation on the seasonal scale, and the similarity in their relative responses on the interannual scale. This relationship has been noted 

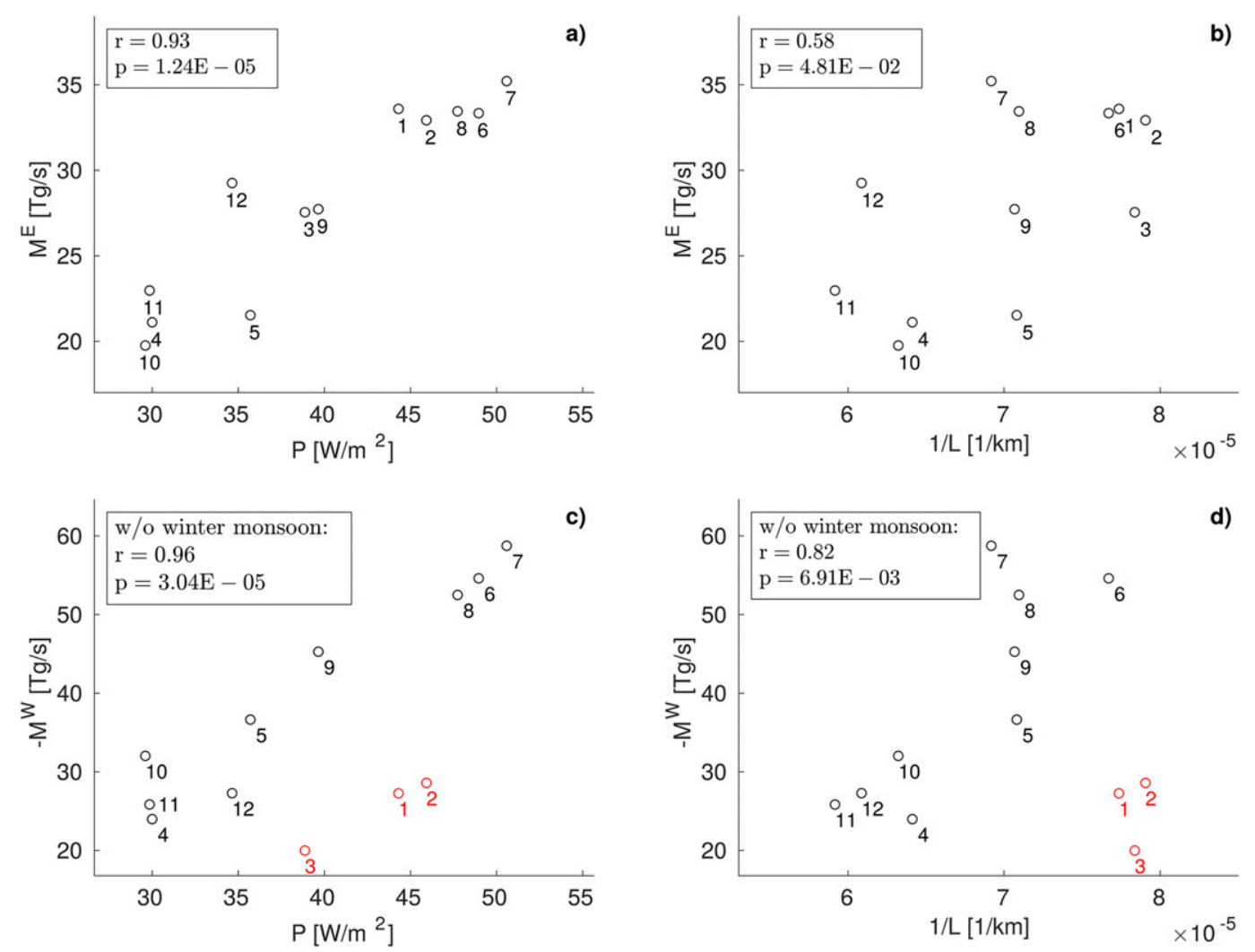

FIG. 7. Streamfunction maximum for the eastern (clockwise) Walker cell as a function of (a) spatial-mean precipitation and (b) reciprocal of the zonal width of the heating $(L)$. Numbers indicate months. All meridional averages are computed from $30^{\circ} \mathrm{S}$ to $30^{\circ} \mathrm{N}$. The linear correlation $(r)$ and its $p$ value $(p)$ are shown in the text boxes. (c),(d) As in (a) and (b), but for the western (counterclockwise) cell. Red colors indicate the Asian winter monsoon season (see text for details).

before; as shown by Merlis and Schneider (2011), it can be derived from a moisture budget analysis, should the atmospheric saturation specific humidity show little seasonal or interannual variability. A similar formula is also obtained from the dry static energy budget [e.g., Eq. $\left(2.10^{\prime}\right)$ of Neelin and Held 1987], if convective heating is the dominant driving term and variability in dry static stability is small. Equation (13) should not be interpreted as a causality, in which the increased precipitation results in a stronger circulation. It is instead a relationship between two coupled variables, whereby the circulation and convective heating behave in a way in which large values of $M$ are associated with large values of $P$ and vice versa.

\section{Discussion and conclusions}

In this study, we derive a linear streamfunction equation for the Walker circulation given specified convective heating, and solve it analytically for a simple heating distribution. With the help of these solutions, we examine the response of the Walker circulation to changes in the zonal or the vertical extent of the heating. The results show that the circulation response strongly depends on the behavior of the total atmospheric heating as a function of scale. If atmospheric heating is directly proportional to scale, increasing the zonal or vertical extent of the heating is associated with a stronger circulation. Conversely, if the total heating remains constant, an increase in scale is associated with a weaker circulation. Similar behavior is observed in the numerical simulations and in the Gill (1980) model, where the circulation responses always share the same sign with the analytic solutions.

The relevance of our conclusions is also evident in the observations. Seasonal and interannual variability in the IndoPacific Walker circulation is tightly coupled to rainfall over the warm pool. On the seasonal scale, the spatial-mean precipitation anomaly is strongly correlated with the circulation strength in both the clockwise (eastern) and the anticlockwise (western) cells (with the exception of the Asian winter monsoon impacting the circulation in the west). Larger zonal scale of the deep convective region is associated with weaker circulation, implying that the atmosphere behaves in a manner analogous to our constant total heating case. Interannual variability behaves similarly; stronger circulation is observed during La Niña, when the spatial-mean precipitation anomaly is large, and the convective region shrinks. The analytic solutions are shown to be most consistent with these observations when strong damping is used. In this strongly damped limit, the solution reduces to a simple linear relationship between the spatial-mean precipitation and circulation strength. 

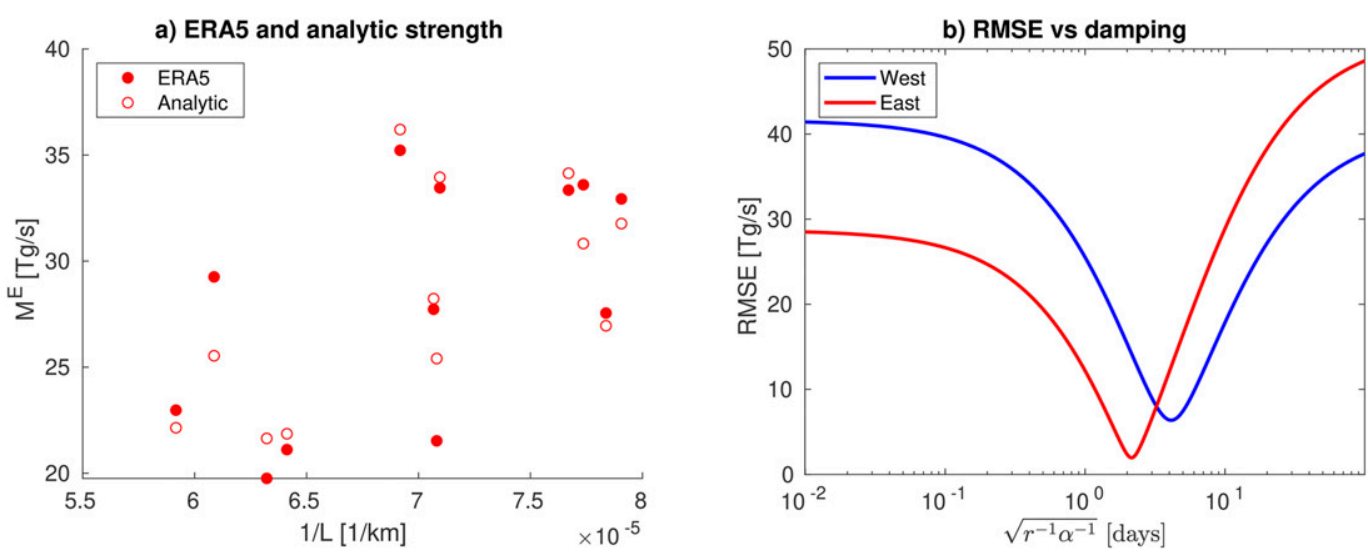

FIG. 8. (a) Comparison of analytic solutions [open circles; Eq. (12) with $\sqrt{r^{-1} \alpha^{-1}}=2$ days or $\Lambda=1.9 \times 10^{-7}$ ] forced with ERA5 precipitation and observed ERA5 eastern-cell mass transports (solid circles) (in the same format as Fig. 7b). (b) The root-mean-square error between the analytic solution and ERA5 observations in the western (blue) and the eastern (red) cell as the damping time scale is varied. See text for details.

These results are mostly in line with previous numerical studies. Geisler (1981) concludes that the circulation gets stronger with increasing scale, when he changes the total heating in a manner consistent with our domain-proportional case. Similarly, Wu et al. (2000b) find that the strength increases with decreasing scale when they hold the total convective heating constant. The streamfunction equation we derived is very similar to the Kuo-Eliassen equation, being a Poisson's equation forced by a gradient of diabatic heating. Likewise, there is a parameter determining the aspect ratio of the response, with the Rayleigh damping in our model being analogous to inertial stability in the Kuo-Eliassen framework.

Damping time scales on the order of days are required to obtain a realistic circulation. This time scale is in line with previous linear models of tropical circulation (e.g., Matsuno 1966; Gill 1980; Webster 1972; Zebiak and Cane 1987), and consistent with observations of convective momentum transport and advection (Lin et al. 2008). However, it contradicts an observational study by Stechmann and Ogrosky (2014), who conclude that the zonal overturning circulation instead resembles an undamped wave response. The underlying cause of this discrepancy is not clear to us, but our damping estimate is quite sensitive to the total diabatic heating. Should the total precipitation be an overestimate of the total heating, longer damping time scales like those of 10 days or more are possible. Another key difference between our models is the fact that Stechmann and Ogrosky (2014) consider the Walker circulation to emerge due to the action of Kelvin and Rossby waves, thus being fundamentally dependent on the rotation of Earth. Although we ignore rotation entirely in the derivation of the analytic solution, our results using the Gill (1980) and the numerical models would indicate that the nonrotating solution captures the first-order behavior of the circulation response. Our numerical model is also consistent with the stronger damping rates in terms of the circulation strength. Some improvements in the analytic solutions could also be made by the inclusion of boundary effects or by switching to a more realistic shape of the heating distribution-however, neither our or the
Stechmann and Ogrosky (2014) model seem to support a very short damping time scale of less than a day.

It might be tempting to apply these conclusions to the study of climate change, when the tropical central and eastern Pacific warm (e.g., Collins et al. 2010) and the convective clouds grow taller (e.g., Hartmann and Larson 2002). However, the robust weakening of the zonal overturning circulation in models is strongly driven by a change in atmospheric static and grossmoist stability (e.g., Knutson and Manabe 1995; Sohn et al. 2016; Wills et al. 2017). Together with changing stability, the convective heating increases to balance a change in atmospheric radiative cooling (e.g., Held and Soden 2006). Thus, there are potential competing effects between stability and the shape and magnitude of diabatic heating, which we do not consider in this study of unforced internal variability.

Future studies need not restrict themselves solely to these analytic solutions. The streamfunction equation can be used directly with an arbitrary shape of convective heating, provided that the solutions are computed numerically. This way, the streamfunction could be obtained for more realistic convective heating shapes, like those output from a numerical model or inferred from observations. Combining these realistic fields with the simple streamfunction equation could pave the way toward deeper understanding of tropical circulations.

Acknowledgments. Juho Iipponen would like to thank the Emil Aaltonen Foundation for their generous support. The authors also greatly acknowledge Dr. Levi Silvers for the many insightful discussions and comments. Observational data have been generated using Copernicus Climate Change Service information (2019).

\section{REFERENCES}

Bayr, T., D. Dommenget, T. Martin, and S. B. Power, 2014: The eastward shift of the Walker circulation in response to global warming and its relationship to ENSO variability. Climate Dyn., 43, 2747-2763, https://doi.org/10.1007/ s00382-014-2091-y. 
Bjerknes, J., 1969: Atmospheric teleconnections from the equatorial Pacific. Mon. Wea. Rev., 97, 163-172, https://doi.org/ 10.1175/1520-0493(1969)097<0163:ATFTEP $>2.3$.CO;2.

Bretherton, C. S., P. N. Blossey, and C. R. Jones, 2013: Mechanisms of marine low cloud sensitivity to idealized climate perturbations: A single-LES exploration extending the CGILS cases. J. Adv. Model. Earth Syst., 5, 316-337, https://doi.org/10.1002/ jame.20019.

Collins, M., and Coauthors, 2010: The impact of global warming on the tropical Pacific Ocean and El Niño. Nat. Geosci., 3, 391397, https://doi.org/10.1038/ngeo868.

Copernicus Climate Change Service, 2017: ERA5: Fifth generation of ECMWF atmospheric reanalyses of the global climate. Copernicus Climate Change Service Climate Data Store, accessed 15 August 2019, https://cds.climate.copernicus.eu/ cdsapp\#!/home.

Eliassen, A., 1951: Slow thermally or frictionally controlled meridional circulation in a circular vortex. Astrophys. Nor., 5, 19-60.

Emanuel, K. A., J. D. Neelin, and C. S. Bretherton, 1994: On large-scale circulations in convecting atmospheres. Quart. J. Roy. Meteor. Soc., 120, 1111-1143, https://doi.org/ 10.1002/qj.49712051902.

Frierson, D. M., 2007: The dynamics of idealized convection schemes and their effect on the zonally averaged tropical circulation. J. Atmos. Sci., 64, 1959-1976, https://doi.org/10.1175/ JAS3935.1.

Furman, M. A., 1994: Compact complex expressions for the electric field of two-dimensional elliptical charge distributions. Amer. J. Phys., 62, 1134-1140, https://doi.org/10.1119/1.17674.

Geisler, J., 1981: A linear model of the Walker circulation. J. Atmos. Sci., 38, 1390-1400, https://doi.org/10.1175/15200469(1981)038<1390:ALMOTW>2.0.CO;2.

Gill, A. E., 1980: Some simple solutions for heat-induced tropical circulation. Quart. J. Roy. Meteor. Soc., 106, 447-462, https:// doi.org/10.1002/qj.49710644905.

Gonzalez, A. O., and G. M. Rojas, 2014: Balanced dynamics of deep and shallow Hadley circulations in the tropics. J. Adv. Model. Earth Syst., 6, 777-804, https://doi.org/ 10.1002/2013MS000278.

Hartmann, D. L., and K. Larson, 2002: An important constraint on tropical cloud-climate feedback. Geophys. Res. Lett., 29, 1951, https://doi.org/10.1029/2002GL015835.

— , H. H. Hendon, and R. A. Houze Jr., 1984: Some implications of the mesoscale circulations in tropical cloud clusters for large-scale dynamics and climate. J. Atmos. Sci., 41, 113-121, https://doi.org/10.1175/1520-0469(1984)041<0113:SIOTMC> 2.0.CO;2.

He, J., and B. J. Soden, 2015: Anthropogenic weakening of the tropical circulation: The relative roles of direct $\mathrm{CO}_{2}$ forcing and sea surface temperature change. J. Climate, 28, 8728-8742, https://doi.org/10.1175/JCLI-D-15-0205.1.

Held, I. M., and M. J. Suarez, 1994: A proposal for the intercomparison of the dynamical cores of atmospheric general circulation models. Bull. Amer. Meteor. Soc., 75, 1825-1830, https:// doi.org/10.1175/1520-0477(1994)075<1825:APFTIO>2.0.CO;2.

— cycle to global warming. J. Climate, 19, 5686-5699, https:// doi.org/10.1175/JCLI3990.1.

Holton, J. R., and D. E. Colton, 1972: A diagnostic study of the vorticity balance at $200 \mathrm{mb}$ in the tropics during the northern summer. J. Atmos. Sci., 29, 1124-1128, https://doi.org/10.1175/ 1520-0469(1972)029<1124:ADSOTV>2.0.CO;2.
Klein, S. A., and D. L. Hartmann, 1993: The seasonal cycle of low stratiform clouds. J. Climate, 6, 1587-1606, https://doi.org/ 10.1175/1520-0442(1993)006<1587:TSCOLS >2.0.CO;2.

Knutson, T. R., and S. Manabe, 1995: Time-mean response over the tropical Pacific to increased $\mathrm{CO}_{2}$ in a coupled oceanatmosphere model. J. Climate, 8, 2181-2199, https://doi.org/ 10.1175/1520-0442(1995)008<2181:TMROTT>2.0.CO;2.

Kuo, H., 1956: Forced and free meridional circulations in the atmosphere. J. Meteor., 13, 561-568, https://doi.org/10.1175/ 1520-0469(1956)013<0561:FAFMCI > 2.0.CO;2.

Lin, J.-L., B. E. Mapes, and W. Han, 2008: What are the sources of mechanical damping in Matsuno-Gill-type models? J. Climate, 21, 165-179, https://doi.org/10.1175/2007JCLI1546.1.

Lutsko, N. J., 2018: The response of an idealized atmosphere to localized tropical heating: Superrotation and the breakdown of linear theory. J. Atmos. Sci., 75, 3-20, https://doi.org/ 10.1175/JAS-D-17-0192.1.

Matsuno, T., 1966: Quasi-geostrophic motions in the equatorial area. J. Meteor. Soc. Japan, 44, 25-43, https://doi.org/10.2151/ jmsj1965.44.1_25.

Merlis, T. M., and T. Schneider, 2011: Changes in zonal surface temperature gradients and Walker circulations in a wide range of climates. J. Climate, 24, 4757-4768, https://doi.org/10.1175/ 2011JCLI4042.1.

Myers, T. A., and J. R. Norris, 2013: Observational evidence that enhanced subsidence reduces subtropical marine boundary layer cloudiness. J. Climate, 26, 7507-7524, https://doi.org/ 10.1175/JCLI-D-12-00736.1.

Neelin, J. D., 2007: Moist dynamics of tropical convection zones in monsoons, teleconnections, and global warming. The Global Circulation of the Atmosphere, T. Schneider and A. Sobel, Eds., Princeton University Press, 267-301.

- , and I. M. Held, 1987: Modeling tropical convergence based on the moist static energy budget. Mon. Wea. Rev., 115, 3-12, https://doi.org/10.1175/1520-0493(1987)115<0003:MTCBOT> 2.0.CO;2.

_ , and J.-Y. Yu, 1994: Modes of tropical variability under convective adjustment and the Madden-Julian oscillation. Part I: Analytical theory. J. Atmos. Sci., 51, 1876-1894, https://doi.org/ 10.1175/1520-0469(1994)051<1876:MOTVUC>2.0.CO;2.

Raymond, D. J., S. L. Sessions, A. H. Sobel, and Ž. Fuchs, 2009: The mechanics of gross moist stability. J. Adv. Model. Earth Syst., 1, 9, https://doi.org/10.3894/JAMES.2009.1.9.

Schubert, W. H., and B. D. McNoldy, 2010: Application of the concepts of Rossby length and Rossby depth to tropical cyclone dynamics. J. Adv. Model. Earth Syst., 2, 7, https://doi.org/ 10.3894/JAMES.2010.2.7.

Schumacher, C., R. A. Houze Jr., and I. Kraucunas, 2004: The tropical dynamical response to latent heating estimates derived from the TRMM Precipitation Radar. J. Atmos. Sci., 61, 1341-1358, https://doi.org/10.1175/1520-0469(2004)061<1341: TTDRTL $>2.0 . \mathrm{CO} ; 2$.

Schwendike, J., P. Govekar, M. J. Reeder, R. Wardle, G. J. Berry, and C. Jakob, 2014: Local partitioning of the overturning circulation in the tropics and the connection to the Hadley and Walker circulations. J. Geophys. Res. Atmos., 119, 1322-1339, https://doi.org/10.1002/2013JD020742.

—, G. J. Berry, M. J. Reeder, C. Jakob, P. Govekar, and R. Wardle, 2015: Trends in the local Hadley and local Walker circulations. J. Geophys. Res. Atmos., 120, 7599-7618, https://doi.org/10.1002/2014JD022652.

Sohn, B.-J., S. Lee, E.-S. Chung, and H.-J. Song, 2016: The role of the dry static stability for the recent change in the Pacific 
Walker circulation. J. Climate, 29, 2765-2779, https://doi.org/ 10.1175/JCLI-D-15-0374.1.

Stechmann, S. N., and H. R. Ogrosky, 2014: The Walker circulation, diabatic heating, and outgoing longwave radiation. Geophys. Res. Lett., 41, 9097-9105, https://doi.org/10.1002/ 2014 GL062257.

Tao, W.-K., and Coauthors, 2016: TRMM latent heating retrieval: Applications and comparisons with field campaigns and largescale analyses. Multiscale Convection-Coupled Systems in the Tropics, Meteor. Monogr., No. 56, Amer. Meteor. Soc., https:// doi.org/10.1175/AMSMONOGRAPHS-D-15-0013.1.

Tarshish, N., N. Jeevanjee, and D. Lecoanet, 2018: Buoyant motion of a turbulent thermal. J. Atmos. Sci., 75, 3233-3244, https:// doi.org/10.1175/JAS-D-17-0371.1.

Trenberth, K. E., D. P. Stepaniak, and J. M. Caron, 2000: The global monsoon as seen through the divergent atmospheric circulation. J. Climate, 13, 3969-3993, https://doi.org/10.1175/ 1520-0442(2000)013<3969:TGMAST>2.0.CO;2.

Vecchi, G. A., B. J. Soden, A. T. Wittenberg I. M. Held, A. Leetmaa, and M. J. Harrison, 2006: Weakening of tropical Pacific atmospheric circulation due to anthropogenic forcing. Nature, 441, 73-76, https://doi.org/10.1038/nature04744.
Webster, P. J., 1972: Response of the tropical atmosphere to local, steady forcing. Mon. Wea. Rev., 100, 518-541, https://doi.org/ 10.1175/1520-0493(1972)100<0518:ROTTAT >2.3.CO;2.

Wills, R. C., X. J. Levine, and T. Schneider, 2017: Local energetic constraints on Walker circulation strength. J. Atmos. Sci., 74, 1907-1922, https://doi.org/10.1175/JAS-D-16-0219.1.

Wu, Z., D. S. Battisti, and E. Sarachik, 2000a: Rayleigh friction, Newtonian cooling, and the linear response to steady tropical heating. J. Atmos. Sci., 57, 1937-1957, https://doi.org/10.1175/ 1520-0469(2000)057<1937:RFNCAT>2.0.CO;2.

—_, E. Sarachik, and D. S. Battisti, 2000b: Vertical structure of convective heating and the three-dimensional structure of the forced circulation on an equatorial beta plane. J. Atmos. Sci., 57, 2169-2187, https://doi.org/10.1175/ 1520-0469(2000)057<2169:VSOCHA > 2.0.CO;2.

Yu, J.-Y., C. Chou, and J. D. Neelin, 1998: Estimating the gross moist stability of the tropical atmosphere. J. Atmos. Sci., 55, 1354-1372, https://doi.org/10.1175/1520-0469(1998)055<1354: ETGMSO $>2.0 . \mathrm{CO} ; 2$.

Zebiak, S. E., and M. A. Cane, 1987: A model El Niño-Southern Oscillation. Mon. Wea. Rev., 115, 2262-2278, https://doi.org/ 10.1175/1520-0493(1987)115<2262:AMENO>2.0.CO;2. 